$\underset{P}{\text { ZIBELINE INTERNATIONAL }}$

ISSN: 2521-0874 (Print)

ISSN: 2521-0505 (online)

CODEN : AIMCCO

\title{
EXPONENTIAL STABILITY CONTROL FOR NETWORKED SYSTEMS WITH INTERVAL DISTRIBUTION TIME DELAYS
}

\author{
Hejun Yao, Fushun Yuan \\ School of Mathematics and Statistics, Anyang Normal University 455002 Henan China \\ *Corresponding Author Email: yaohejun@126.com
}

This is an open access article distributed under the Creative Commons Attribution License, which permits unrestricted use, distribution, and reproduction in any medium, provided the original work is properly cited.

\begin{tabular}{l} 
ARTICLE DETAILS \\
\hline Article History: \\
Received 12 December 2017 \\
Accepted 10 January 2018 \\
Available online 19 February 2018 \\
\hline
\end{tabular}

\begin{abstract}
To study the problem of exponential stability control for a class of networked control systems with interval distribution time delays, a new approach is given to model the networked control systems with the stochastic time delays which is assumed to be satisfying a interval Bernoulli distribution. Based on linear matrix inequality approach, the mean-square exponential stability controller design method is presented, and the controller gain matrix is obtain by solving a linear matrix inequality. Moreover, a Lyapunov functional is used, and some stack matrices, which bring much flexibility in solving LMI, are introduced during the proof. A numerical example is given to demonstrate the validity of the results.
\end{abstract}

\section{KEYWORDS}

Networked control systems, Stochastic delays, Linear matrix inequality (LMI)

\section{INTRODUCTION}

Networked control systems (NCSs) are systems where the feedback loop is closed via a communication network in which information, from various components such as sensors, controllers and actuators, is exchanged with limited bandwidth. NCSs have received increasing attentions in recent years due to their low cost simple installation and maintenance and high reliability [1,2].

However, the network itself is dynamic system that exhibits characteristics such as network-induced delays. The delays come from the time sharing of the communication medium as well as the computation time required for physical signal coding and communication processing. As is known, network-induced delays can degrade a system's performance and even cause system instability. Many researchers have studied stability analysis and controller design for NCSs $[3,4]$. It is quite common in practice that the time delays occur in a random way, rather than a deterministic way. Based on a similar Bernoulli stochastic model, study NCSs with both sensor-tocontroller and controller-to-actuator stochastic delays and design the controller gain [5-6]. One step stochastic delays or one stochastic packet dropout is considered in these papers.

The stabilization problem for a networked control system with Markov communication delays existing in both the system state and the mode signal [7]. The problem of the stabilization of NCSs with packet dropout [8]. A networked predictive control method for networked systems with stochastic delay and data dropout is proposed in to compensate the networked-induced delay [9-10]. This paper considers the problem of mean-square exponential stability control for a class of networked control systems with interval distribution time delay. A new approach is given to model the networked control systems. Based on the LIM approach and Lyapunov stability theorem, the mean-square exponential stability condition is given.

\section{PROBLEM FORMULATION}

Consider the following control system with delay

$\dot{x}(t)=A x(t)+A_{d} x(t-d)+B u(t)$

$x(t)=\phi(t) \quad t \in[-d, 0]$ where $x(t) \in R_{n}$ is the state vector, $u(t) \in R_{m}$ is the input vector, $d$ is state delay $A, A_{d} \in \mathrm{R}^{\mathrm{n} \times \mathrm{n}}$ are known real constant matrices, $B \in R^{n \times m}$ is input matrix, $\varphi(t) \in R^{n}$ is given initial state on[-d, 0$]$.

Throughout this note, we suppose that all the system's states are available for a state feedback control. In the presence of the control network, data transfers between the controller and the remote system, e.g., sensors and actuators in a distributed control system will induce network delay in addition to the controller proceeding delay. We introduce stochastic delay $\tau \mathrm{t}()$ to denote the network-induced delay. In this note we make the following assumptions:

Assumption 1: Sensor and controller are clock-driven;

Assumption 2: Actuator is event-driven.

We will design the state feedback controller

$u(t)=K x(t-\tau(t))$

Inserting the controller (2) into system (1), we obtain the closed system:

$$
\begin{aligned}
\dot{x}(t) & =A x(t)+A_{d} x(t-d)+B K x(t-\tau(t)) \\
x(t) & =\psi(t) \quad t \in\left[\begin{array}{ll}
-\bar{d}, & 0
\end{array}\right]
\end{aligned}
$$

The initial condition of the state is supplemented as $x(t)=\psi(t)$, where $\psi$ $(t)$ is a smooth function on $[-\bar{d}, 0], \bar{d}=\max \{\tau, d\}$.Therefore, there exists a positive constant $\bar{\psi}$ satisfying

$\|\dot{\psi}(t)\| \leq \psi \quad \mathrm{t} \in[-\overline{\mathrm{d}}, 0]$

It is assumed that there exists a constant $\tau_{1} \in[\tau, 0]$ such that the probability of $\tau(t)$ taking values in $\left[0, \tau_{1}\right)$ and $\left[\tau_{1}, \tau\right]$ can be observed. In order to employ the information of the probability distribution of the delays, the following sets are proposed firstly

$$
\Omega_{1}=\left\{t: \tau(t) \in\left[0, \tau_{1}\right)\right\}, \Omega_{2}=\left\{t: \tau(t) \in\left[\tau_{1}, \tau\right]\right\}
$$

Obviously, $\Omega_{1} \cup \Omega_{2}=R^{+}$and $\Omega_{1} \cap \Omega_{2}=\Phi$

Then we define two functions as: 


$$
h_{1}(t)=\left\{\begin{array}{ll}
\tau(t) & t \in \Omega_{1} \\
0 & t \notin \Omega_{1}
\end{array}, \quad h_{2}(t)= \begin{cases}\tau(t) & t \in \Omega_{2} \\
\tau_{1} & t \notin \Omega_{2}\end{cases}\right.
$$

Corresponding to $\tau(t)$ taking values in different intervals, a stochastic variable $\beta(t)$ is defined

$$
\beta(t)= \begin{cases}1 & t \in \Omega_{1} \\ 0 & t \in \Omega_{2}\end{cases}
$$

Where we suppose that $\beta(t)$ is a Bernoulli distributed sequence with $\operatorname{Pr}$ $o b\{\beta(t)=1\}=E\{\beta(t)\}=\beta$, where $\beta \in[0,1]$ is a constant.

With (4), (5), we know that the systems (3) is equivalent to

$$
\begin{aligned}
\dot{x}(t) & =A x(t)+A_{d} x(t-d)+\beta(t) B K x\left(t-h_{1}(t)\right)+(1-\beta(t)) B K x\left(t-h_{2}(t)\right) \\
& =\bar{A} \xi(t) \\
x(t) & =\psi(t) \quad t \in[-\bar{d}, 0]
\end{aligned}
$$

where

$$
\begin{aligned}
& \bar{A}=\left[\begin{array}{ll}
A A_{d} \beta(t) B K(1-\beta(t)) B K
\end{array}\right] \\
& \xi^{T}(t)=\left[\begin{array}{ll}
x^{T}(t), & \left.x^{T}(t-d), x^{T}\left(t-h_{1}(t)\right), x^{T}\left(t-h_{2}(t)\right)\right]
\end{array}\right.
\end{aligned}
$$

\section{MAIN RESULTS}

Lemma 1 For any vectors ,ab and matrices $\mathrm{N}, \mathrm{XY}, \mathrm{Z}$ with appropriate dimensions, if the following matrix inequality holds [2]

$\left[\begin{array}{cc}X & Y \\ Y^{T} & Z\end{array}\right] \geq 0$

then we have

$$
-2 a^{T} N b \leq \inf _{X, Y, Z}\left[\begin{array}{l}
a \\
b
\end{array}\right]^{T}\left[\begin{array}{cc}
X & Y-N \\
Y^{T}-N^{T} & Z
\end{array}\right]\left[\begin{array}{l}
a \\
b
\end{array}\right]
$$

Lemma 2 The LMI $\left[\begin{array}{cc}Y(x) & W(x) \\ * & R(x)\end{array}\right]>0$ is equivalent to [4]

$R(x)>0, Y(x)-W(x) R^{-1}(x) W^{\mathrm{T}}(x)>0$

where $Y(\mathrm{x})=Y^{\mathrm{T}}(x), R(x)=R^{\mathrm{T}}(x)$ depend on $\mathrm{x}$.

Theorem 1 For the given constants $\alpha>0,1 \geq \beta \geq 0$, if there exist positivedefinite matrices $P, Q, R \in R^{\mathrm{n} \times \mathrm{n}}$ and matrices $K \in R^{\mathrm{m} \times \mathrm{n}}, X, Y$ with appropriate dimensions, such that the following matrix inequality holds

$\Theta=\left[\begin{array}{cc}\Theta_{11} & \Theta_{12} \\ * & \Theta_{22}\end{array}\right]<0$

where

$$
\begin{aligned}
& \Theta_{11}=\left[\begin{array}{cc}
P A+A^{T} P+Q+2 \alpha P & P \bar{A}_{d}+\tau_{2} X_{12}+\tau_{2} A^{T} R A_{d} \\
+\tau_{2} X_{11}+\tau_{2} A^{T} R A & \\
* & -e^{-2 \alpha d} Q+\tau_{2} X_{22}+\tau_{2} A_{d}^{T} R A_{d}
\end{array}\right] \\
& \Theta_{12}=\left[\begin{array}{cc}
P \beta B K+Y_{1}+\tau_{2} X_{13}+\tau_{2} A^{T} R \beta B K & P(1-\beta) B K+\tau_{2} X_{14}-Y_{1}+\tau_{2} A^{T} R(1-\beta) B K \\
Y_{2}+\tau_{2} X_{23}+\tau_{2} A_{d}^{T} R \beta B K & \tau X_{24}-Y_{2}+\tau_{2} A_{d}^{T} R(1-\beta) B K
\end{array}\right] \text { with the controller } \\
& \Theta_{22}=\left[\begin{array}{cc}
\tau_{2} X_{33}+Y_{3}+Y^{T}+\tau_{2} K^{T} B^{T} R \beta B K & -Y_{3}+Y_{4}^{T}+\tau_{2} X_{34} \\
* & \tau_{2} X_{44}-Y_{4}-Y^{T}+\tau_{2} K^{T} B^{T} R(1-\beta) B K
\end{array}\right]
\end{aligned}
$$

(2), the network control systems (6) is mean-square exponentially stable.

Proof : Choose a Lyapunov functional candidate for the system (6) as follows :

$$
\begin{aligned}
& \dot{V}(t)+2 \alpha V(t) \\
= & 2 x^{T}(t) P \dot{x}(t)+x^{T}(t) Q x(t)-x^{T}(t-d) Q e^{-2 \alpha d} x(t-d) \\
& +\tau \dot{x}^{T}(t) R \dot{x}(t)+2 \alpha x^{T}(t) P x(t)-\int_{t-\tau_{2}}^{t} \dot{x}^{T}(s) R e^{2 \alpha(s-t)} \dot{x}(s) d s
\end{aligned}
$$

With

$x\left(t-h_{1}(t)\right)-x\left(t-h_{2}(t)\right)-\int_{-}^{t-h_{1}(t)} \dot{x}(s) d s=0$
For any $4 \mathrm{n} \times \mathrm{n}$ matrix

$N=\left[\begin{array}{l}N_{1} \\ N_{2} \\ N_{3}\end{array}\right]$

We know

$$
0=\xi^{T}(t) N\left[x\left(t-h_{1}(t)\right)-x\left(t-h_{2}(t)\right)-\int_{t-h_{2}(t)}^{t-h_{1}(t)} \dot{x}(s) d s\right]
$$

With lemma1 and (9), we obtain

$$
\begin{aligned}
0 \leq & 2 \xi^{T}(t) N\left[x\left(t-h_{1}(t)\right)-x\left(t-h_{2}(t)\right)\right] \\
& +\int_{t-h_{2}(t)}^{t-h_{1}(t)}\left[\begin{array}{c}
\xi(t) \\
\dot{x}(s)
\end{array}\right]^{T}\left[\begin{array}{cc}
X & Y-N \\
Y^{T}-N^{T} & R e^{2 \alpha(s-t)}
\end{array}\right]\left[\begin{array}{c}
\xi(t) \\
\dot{x}(s)
\end{array}\right] d s \\
\leq & 2 \xi^{T}(t) Y\left[x\left(t-h_{1}(t)\right)-x\left(t-h_{2}(t)\right)\right]+\tau_{2} \xi^{T}(t) X \xi(t) \\
& +\int_{t-\tau_{2}}^{t} \dot{x}^{T}(s) R e^{2 \alpha(s-t)} \dot{x}(s) d s
\end{aligned}
$$

Inserting (10) into (8), we have :

$$
\begin{aligned}
\dot{V}(t)+2 \alpha V(t) \leq & x^{T}(t)[P A+A P+Q+2 \alpha P] x(t) \\
& +2 x^{T}(t) P A_{d} x(t-d)+2 x^{T}(t) P \beta(t) B K x\left(t-h_{1}(t)\right) \\
& +2 x^{T}(t) P(1-\beta(t)) B K x\left(t-h_{2}(t)\right) \\
& -x^{T}(t-d) Q e^{-2 \alpha d} x(t-d)+2 \xi^{T}(t) Y\left[\begin{array}{llll}
0 & 0 & I & -I
\end{array}\right] \xi(t) \\
& +\tau_{2} \xi^{T}(t) X \xi(t)+\tau_{2} \dot{x}^{T}(t) R \dot{x}(t)
\end{aligned}
$$

where

$$
\dot{x}^{T}(t) R \dot{x}(t)
$$$$
=\tau_{2} \xi^{T}(t)\left[\begin{array}{cccc}
A^{T} R A & A^{T} R A_{d} & A^{T} R \beta(t) B K & A^{T} R(1-\beta(t)) B K \\
* & A_{d}^{T} R A_{d} & A_{d}^{T} R \beta(t) B K & A_{d}^{T} R(1-\beta(t)) B K \\
* & * & \beta^{2}(t) K^{T} B^{T} R B K & \beta(t)(1-\beta(t)) K^{T} B^{T} R B K \\
* & * & * & (1-\beta(t))^{2} K^{T} B^{T} R B
\end{array}\right] \xi(t)
$$

Obviously

$$
2 \xi^{T}(t)\left[\begin{array}{c}
Y_{1} \\
Y_{2} \\
Y_{3} \\
Y_{4}
\end{array}\right]\left[\begin{array}{llll}
0 & 0 & I & -I
\end{array}\right] \xi(t)=\xi^{T}(t)\left[\begin{array}{cccc}
0 & 0 & Y_{1} & -Y_{1} \\
* & 0 & Y_{2} & -Y_{2} \\
* & * & Y_{3}+Y_{3}^{T} & -Y_{3}+Y_{4}^{T} \\
* & * & * & -Y_{4}-Y_{4}^{T}
\end{array}\right] \xi(t)
$$

Inserting (12-13) into (11), we obtain

$$
E\{\dot{V}(t)+2 \alpha V(t)\} \leq \sum \sum \mu(z(t)) \mu(z(t)) \xi^{T}(t) \Theta \xi(t)
$$

With matrix inequality（7）, we know

$\{E \dot{V}(t)\}<-2 \alpha\{E V(t)\}$

therefore

$\{E V(t)\}<\{E V(0)\} e^{-\alpha t} \leq\left[\lambda_{\max }(P)+d \lambda_{\max }(Q)+\tau \lambda \max (R) \psi^{2}\right] E\left\{\left.\|\psi \psi(t)\|\right|^{2}\right\} e^{-2 \alpha t}$

Obviously

$$
E\{V(t)\} \geq \lambda \quad(P) E\left\{\|x(t)\|^{2}\right\}
$$

From (14-15), we obtain

$$
E\{\|x(t)\|\}<\sqrt{\frac{\lambda_{\max }(P)+d \lambda_{\text {max }}(Q)+\tau \lambda_{\text {max }}(R) \bar{\psi}^{2}}{\lambda_{\text {min }}(P)}} E\{\|\psi(t)\|\} \mathrm{e}^{-\alpha t}
$$

With the Lyapunov stability theorem and the above inequality, we know that the system (6) is mean-square exponentially stable.

Theorem 2 For the given constants $\alpha>0,1 \geq \beta \geq 0$, if there exist positivedefinite matrices $\bar{P}, \bar{Q}, \bar{R} \in R^{n \times n}$ and matrix $\bar{K} \in R^{m \times n}, \bar{X}, \bar{Y}$ with appropriate dimensions, such that the following linear matrix inequality holds

$$
\Xi=\left[\begin{array}{cc}
\Xi_{11} & \Xi_{12} \\
* & \Xi_{22}
\end{array}\right]<0
$$


Where

$$
\begin{gathered}
\Xi_{11}=\left[\begin{array}{ccc}
A \bar{P}+\bar{P} A^{T}+\bar{Q} & A_{d} \bar{P}+\tau_{2} \bar{X}_{12} & \beta B \bar{K}+\tau_{2} \bar{X}_{13}+\bar{Y}_{1} \\
+2 \alpha \bar{P}+\tau_{2} \bar{X}_{11} & -e^{-2 \alpha d} \bar{Q}_{2} \tau_{2} \bar{X}_{22} & \tau_{2} \bar{X}_{23}+\bar{Y}_{2} \\
* & * & \tau_{2} \bar{X}_{33}+\bar{Y}_{3}+\bar{Y}^{T} \\
* & &
\end{array}\right] \\
\Xi_{12}=\left[\begin{array}{ccc}
(1-\beta) B \bar{K}-\bar{Y}_{1}+\tau_{2} \bar{X}_{14} & \tau_{2} \beta \bar{P} A^{T} & \tau_{2}(1-\beta) \bar{P} A^{T} \\
\tau_{2} \bar{X}_{24}-\bar{Y} & \tau_{2} \beta \bar{P} A_{d}^{T} & \tau_{2}(1-\beta) \bar{P} A_{d}^{T} \\
\tau_{2} \bar{X}_{34}+\bar{Y}_{4}^{T}-\bar{Y}_{3} & \tau_{2} \beta \bar{K}^{T} B^{T} & 0
\end{array}\right] \\
\Xi_{12}=\left[\begin{array}{ccc}
\tau_{2} \bar{X}_{44}-\bar{Y}_{4}-\bar{Y}^{T} & 0 & \tau_{2}(1-\beta) \bar{K}^{T} B^{T} \\
-\tau_{2} \beta \bar{R} & 0
\end{array}\right]
\end{gathered}
$$

with the controller $u(t)=\overline{K P^{-1}} x(t)$, the systems (6) is mean-square exponentially stable.

Proof

$$
\Theta=\Theta_{0}+\alpha_{1}^{T} \tau_{2} \beta R^{-1} \alpha_{1}+\alpha_{2}^{T} \overline{\tau_{2}(1-\beta)} \quad R_{2}^{-1} \alpha
$$

where

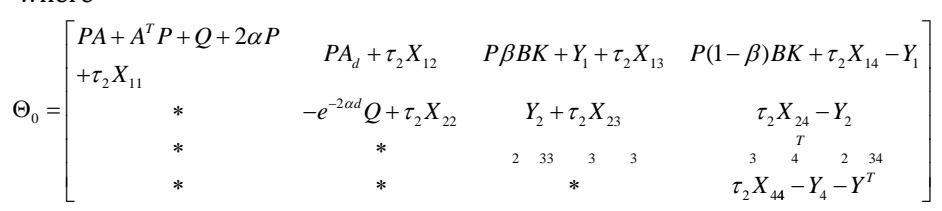

$\alpha_{1}=\left[\begin{array}{llll}\tau_{2} \beta R A & \tau_{2} \beta R A_{d} & \tau_{2} \beta R B K & 0\end{array}\right]$

$\alpha_{2}=\left[\begin{array}{llll}\tau_{2}(1-\beta) R A & \tau_{2}(1-\beta) R A_{d} & 0 & \tau_{2}(1-\beta) R B K\end{array}\right]$

With lemma2, we know that inequality $\Theta<0$ is equivalent to

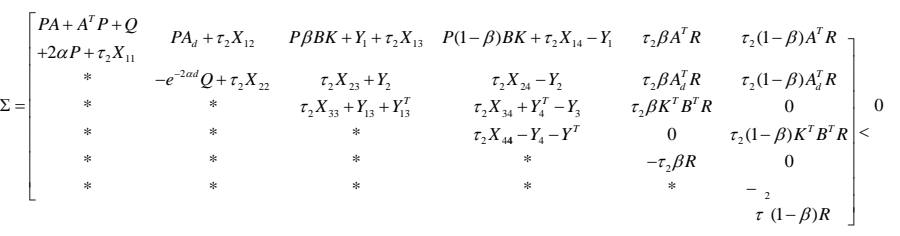

Pre- and Post-multiplying the inequality (17) by $\operatorname{diag}\left\{P^{-1} P^{-1} P^{-1} P^{-1} R^{-1} R^{-1}\right\}$, and giving some transformations:

$$
\bar{P}=P^{-1} \quad \bar{Q}=P^{-1} \mathrm{Q} P^{-1} \quad \bar{K}=K P^{-1} \quad \bar{X}=P^{-1} X P^{-1} \quad \bar{Y}=P^{-1} Y P^{-1} \quad \bar{R}=R^{-1}
$$

we know that inequality $\Sigma<0$ is equivalent to (16). Therefore, the linear matrix inequality (16) is equivalent to (7). With Theorem1, the systems (6) is mean-square exponentially stable.

\section{SIMULATION}

Consider the networked control systems in the form of (7), where

$A=\left[\begin{array}{cc}-3 & 1 \\ 0 & -4\end{array}\right], A_{d}=\left[\begin{array}{cc}-1 & 0 \\ 1 & -2\end{array}\right], B=\left[\begin{array}{l}0.2 \\ 0.1\end{array}\right], \tau=1, \tau_{1}=0.5, \beta=0.5$,

$\alpha=0.1, d=0.1$. Solving the linear matrix inequality (16), we can obtain the gain matrix $K$ of the stabilizing controller $u(t)$

$$
K=\left[\begin{array}{ll}
-0.7645 & 2.5692
\end{array}\right]
$$

From the theorem 2, we know that the systems (6) is mean-square exponentially stable.

\section{CONCLUSION}

This paper considers the problem of mean-square exponential stability control for a class of networked control systems with interval distribution time delay. Based on the Lyapunov stability theorem, a sufficient condition and the controller design approach are given in term of LMI.

\section{ACKNOWLEDGMENTS}

The author would like to thank the associate editor and the anonymous reviewers for their constructive comments and suggestions to improve the quality and the presentation of the paper. This work was supported by National Nature Science Foundation under Project (61073065); Nature Science Foundation of Henan Province under Grant 092300410145; Henan Province Science and Technology Key Project (172102210162); The National College Students' Innovative Entrepreneurial Training Project (201610479045); The Education Department of Henan Province Key Foundation under Grant (18B110001), Anyang Normal University Innovation Foundation Project under Grant (ASCX/2017-Z102).

\section{REFERENCES}

[1] Xia, Y., Fu, M., Liu, B., Liu, G. 2009. Design and Performance Analysis of Networked Control Systems with Random Delay. Journal of Systems Engineering and Electronics, 20 (2), 807-822.

[2] Xia, X., Zhang, D., Zheng, L., Zheng, N. 2008. Modeling and Stabilization for a Class of Nonlinear Networked Control Systems, a T-S Fuzzy Approach. Progress in Natural Science, 18 (2), 1031-1037.

[3] Zhang, H., Yang, J., Su, C. 2007. T-S Fuzzy Model Based Robust Hœ Design for Networked Control Systems with Uncertainties. IEEE Transactions on Industrial Informatics, 3 (2), 289-301.

[4] Jiang, X., Han, Q. 2008. On Designing Fuzzy Controllers for a Class of Nonlinear Networked Control Systems. IEEE Transactions on Fuzzy Systems, 16 (2), 1050-1060.

[5] Schenato, L. 2006. Optimal Estimation in Networked Control Systems Subject to Random Delay and Packet Loss. Proceedings of the 45th IEEE Conference on Decision and Control, San Diego, CA, USA, 5615-5620.

[6] Li, J.G., Yuan, J.Q., Lu, J.G. 2010. Observer-based Ho Control for Networked Nonlinear Systems with Random Packet Loss. ISA Transactions, 49 (1), 39-46.

[7] Liu, M., Hoa, D.W. 2009. Stabilization of Markov Jump Linear System Over Networks with Random Communication Delay. Automatica, 45 (2), 416-421.

[8] Xiong, J.L., Lam, J. 2007. Stabilization of Linear Systems Over Networks with Bounded Packet Loss. Automatica, 43 (2), 80-87.

[9] Zhao, Y., Liu, G., Rees, D. 2008. A Predictive Control Based Approach to Networked Wiener Systems. International Journal of Innovative Computing, Information and Control, 4 (2), 2793-2802.

[10] Xia, Y., Liu, G.P., Fu, M., Rees, D. 2009. Predictive Control of Networked Systems with Random Delay and Data Dropout. IET Control Theory \& Applications, 3 (2), 1476-1486. 